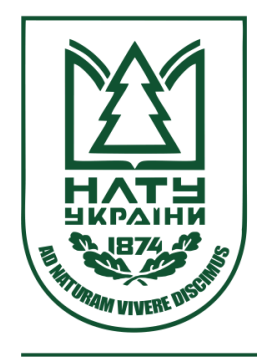

Науковий вісник НЛТУ України

Scientific Bulletin of UNFU

http://nv.nltu.edu.ua

https://doi.org/10.36930/40291013

Article received 15.10.2019 p.

Article accepted 26.12.2019 p.

удк 532.54.013.2

ISSN 1994-7836 (print)

ISSN 2519-2477 (online)

$@ \bowtie$ Correspondence author

R. M. Hnativ

gnativ.roman.m@gmail.com

\title{
МАТЕМАТИЧНА МОДЕЛЬ ДЛЯ ДОСЛІДЖЕННЯ НЕСТАЦІОНАРНИХ ТЕЧІЙ НЕСТИСЛИВОЇ РІДИНИ У ТРУБАХ
}

\begin{abstract}
Проаналізовано наукові роботи з розв'язку задач про нестаціонарний рух рідини в циліндричних трубах. Встановлено, що під час розв'язування задач неусталених рухів рідини у трубах виникає потреба визначення швидкостей рідини у перерізах трубопроводу, як в осьовому, так і радіальному напрямках. Класичні методи вирішення цієї задачі не дають задовільних результатів. Удосконалено методику розрахунку нестаціонарних потоків рідини на основі дисипативної моделі. У дослідженнях використано модель із врахуванням дисипативних процесів течії в'язкої рідини, яку вивчали варіаційним методом, враховуючи початкові і граничні умови. Об'єктом дослідження є гідравлічні процеси в неусталених потоках в'язкої рідини у циліндричному трубопроводі. Запропоновано удосконалену методику розрахунку неусталених потоків для нестисливої рідини на основі дисипативної моделі. З'ясовано, що в цьому випадку припущення про нехтування компонентою радіальної швидкості є асимптотично обгрунтованим. Наведено низькочастотні розв'язки рівнянь Нав'є-Стокса для спрощеної моделі нестисливої рідини. Дисипативна модель грунтується на двох припущеннях про порядки розв'язків рівнянь Нав'є-Стокса стосовно часу та осьової координати. При цьому ніякі припущення щодо порядку величини компонентів швидкості не виво-
\end{abstract} дяться.

Ключові слова: неусталений; нестаціонарний; рух рідини; дисипативна модель; розподіл швидкостей.

Вступ. Під час розв'язку задач неусталених рухів рідини у трубах виникає потреба визначення швидкостей рідини в перерізах трубопроводу як в осьовому, так i радіальному напрямках. Класичні методи вирішення цієї задачі не дають задовільних результатів.

Перші теоретичні дослідження нестаціонарних течій нестисливої рідини в трубах, де використовують диференціальні рівняння, що описують зміни профілю швидкості і коефіцієнта тертя, проводили вже наприкінці XIX ст. (Gromeka, 1952). Модель в'язкої нестисливої рідини має широке застосування до сьогодні.

Вивчення нестаціонарних течій стисливої рідини в трубах із змінним коефіцієнтом тертя почалося тільки в 50-ті роки XX ст. При цьому застосування знайшли дві моделі - модель плоскопаралельної течії (Shablovskii, 2007; Mochalin, 2002; Jayasinghe, \& Leutheusser, 1972) i дисипативна модель (Magrakvelidze, 2005; D'Suza, \& Oldenburger, 1964; Barmetov, \& Palishkin, 2007; Bondarenko, \& Terentev, 2009; Charnyi, 1975; Popov, 1977). Хоча модель плоскопаралельної течії є простішою, широкого застосування вона не знайшла, оскільки вона не може бути теоретично обгрунтована.
Під час виведення дисипативної моделі з рівнянь Нав'є-Стокса застосовували метод, за яким поступово вводиться низка припущень і на основі їх виводиться відповідна спрощена система диференціальних рівнянь (Magrakvelidze, 2005; D'Suza, \& Oldenburger, 1964; Barmetov, \& Palishkin, 2007; Bondarenko, \& Terentev, 2009; Charnyi, 1975; Popov, 1977). За такого висновку залишається незрозумілою "замкнутість" зроблених припущень і отриманої моделі, а також зв'язки між введеними припущеннями.

На основі грунтовних досліджень отримано характеристики розповсюдження збурень у циліндричних трубах за рівняннями Нав'є-Стокса для окремих випадків при певних додаткових припущеннях (Adamkowski, \& Lewandowski, 2006; Rakhmatullin, \& Kim, 2006; Girgidov, 2009; Gnativ, \& Mikitin, 2012; Gnativ, 2013). У цих роботах головна увагу приділено вивченню характеристики поширення збурень, а не розробленню та обгрунтуванню відповідних спрощених моделей.

Об'єкт і методи дослідження. Застосовуючи останній метод, у цій роботі проведено систематичне виведення й обгрунтування дисипативної моделі. Для цього

Інформація про авторів:

Яхно Олег Михайлович, д-р техн. наук, професор, кафедра прикладної гідроаеромеханіки і механотроніки. Email: oleg.yakhno@gmail.com; https://orcid.org/0000-0002-9522-5549

Гнатів Роман Маріянович, д-р техн. наук, доцент, кафедра гідравліки та сантехники. Email: gnativ.roman.m@gmail.com; https://orcid.org/0000-0002-4931-7493

Гнатів Iгор Романович, аспірант, кафедра екологіï. Email: gnativ13@gmail.com; https://orcid.org/0000-0002-2987-1673

Цитування за ДСтУ: Яхно О. М., Гнатів Р. М., Гнатів І. Р. Математична модель для дослідження нестаціонарних течій нестисливої рідини у трубах. Науковий вісник НЛтУ України. 2019, т. 29, № 10. С. 71-74.

Citation APA: Yahno, O. M., Hnativ, R. M., \& Hnativ, I. R. (2019). Mathematical model for the study of unsteady flows of an incompressible fluid in pipes. Scientific Bulletin of UNFU, 29(10), 71-74. https://doi.org/10.36930/40291013 
на основі лінеаризованих рівнянь Нав'є-Стокса для стисливої рідини знаходяться елементарні розв'язки цих рівнянь, що відповідають хвильовим рухам рідини в напрямку осі труби. Ввівши певні припущення про порядки фізичних величин і параметри розв'язків та застосовуючи асимптотичний аналіз, проводяться спрощення цих елементарних розв'язків. Наближені розв'язки, що отримуються внаслідок такого аналізу, використовуються як елементарні розв'язки відповідної дисипативної моделі. Стає зрозумілим, що дисипативна модель грунтується на двох припущеннях про порядки розв'язків рівнянь Нав'є-Стокса стосовно часу та осьової координати. При цьому ніякі припущення щодо порядку величини компонентів швидкості не виводяться.

Результати дослідження. Розглянемо випадки нестисливої рідини і виведемо для неї математичну модель для опису низькочастотних розв'язків, виходячи з лінеаризованих рівнянь Нав'є-Стокса і нерозривності для осесиметричних рухів:

$$
\begin{aligned}
& \rho \frac{\partial v_{z}}{\partial t}=-\frac{\partial p}{\partial z}+\mu \nabla^{2} V_{z}+\frac{1}{3} \mu \frac{\partial \theta}{\partial z}, \\
& \rho \frac{\partial v_{r}}{\partial t}=-\frac{\partial p}{\partial r}+\mu\left(\nabla^{2} v_{r}-\frac{v_{r}}{r^{2}}\right)+\frac{1}{3} \mu \frac{\partial \theta}{\partial r}, \\
& \rho c^{2} \theta+\frac{\partial p}{\partial t}=0, \\
& \theta=\frac{\partial v_{r}}{\partial r}+\frac{v_{r}}{r}+\frac{\partial v_{z}}{\partial z}, \\
& \nabla^{2}=\frac{\partial^{2}}{\partial r^{2}}+\frac{1}{r} \frac{\partial}{\partial r}+\frac{\partial^{2}}{\partial z^{2}},
\end{aligned}
$$

де: $v_{z}, v_{r}$ - складові швидкості в напрямку осей $z, r ; p-$ тиск; $\rho-$ густина рідини; $\mu$ - коефіцієнт в'язкості; $c-$ швидкість звуку в рідині; $t$ - час.

Перейдемо до безрозмірних координат $\xi, \eta, \tau$ та безрозмірних змінних $u_{\xi}, u_{\eta}, \vartheta, q$ так:

$$
\begin{gathered}
\xi=\frac{z}{R}, \eta=\frac{r}{R}, \tau=\frac{c}{R} t, \\
u_{\xi}=\frac{v_{z}}{U}, u_{\eta}=\frac{v_{r}}{U}, \vartheta=\frac{R \theta}{U}, q=\frac{1}{c p U} p,
\end{gathered}
$$

де: $R$ - радіус труби; $U$ - нормуюча швидкість.

У нових змінних рівняння (1)-(3) і співвідношення (4), (5) набувають вигляду:

$$
\begin{gathered}
\beta \frac{\partial u_{\xi}}{\partial \tau}=-\beta \frac{\partial q}{\partial \xi}+\tilde{\nabla}^{2} u_{\xi}+\frac{1}{3} \frac{\partial \vartheta}{\partial \xi}, \\
\beta \frac{\partial u_{\eta}}{\partial \tau}=-\beta \frac{\partial q}{\partial \eta}+\tilde{\nabla}^{2} u_{\eta}-\frac{u_{\eta}}{\eta^{2}}+\frac{1}{3} \frac{\partial \vartheta}{\partial \eta}, \\
\vartheta+\frac{\partial q}{\partial \tau}=0, \\
\vartheta=\frac{\partial u_{\eta}}{\partial \eta}+\frac{u_{\eta}}{\eta}+\frac{\partial u_{\xi}}{\partial \xi}, \\
\tilde{\nabla}^{2}=\frac{\partial^{2}}{\partial \eta^{2}}+\frac{1}{\eta} \frac{\partial}{\partial \eta}+\frac{\partial^{2}}{\partial \xi^{2}}, \\
\beta=\frac{c R}{v}, v=\frac{\mu}{\rho} .
\end{gathered}
$$

Розглянемо частинні розв'язки системи (8)-(11) у вигляді:

$$
\begin{gathered}
u_{\xi}=U_{\xi}(\eta) e^{i(\omega \tau-k \xi)}, u_{\eta}=U_{\eta}(\eta) e^{i(\omega \tau-k \xi)}, \vartheta=\theta(\eta) e^{i(\omega \tau-k \xi)}, \\
q=Q(\eta) e^{i(\omega \tau-k \xi)} .
\end{gathered}
$$

Підставляючи вираз (13) в рівняння (8)-(11), отримуємо для визначення функцій $U_{\xi}, U_{\eta}, \theta, Q$ систему:

$$
\begin{gathered}
\left(\tilde{\nabla}^{2}-i \omega \beta\right) U_{\xi}+i k \beta Q-\frac{1}{3} i k \theta=0, \\
\tilde{\nabla}^{2} U_{\eta}-\left(\frac{1}{\eta^{2}}+i \omega \beta\right) U_{\eta}-\beta \frac{d Q}{d \eta}+\frac{1}{3} \frac{d \theta}{d \eta}=0, \\
\theta+i \omega Q=0, \\
\theta=\frac{d U_{\eta}}{d \eta}+\frac{1}{\eta} U_{\eta}-i k U_{k} .
\end{gathered}
$$

Введемо таке позначення

$$
\tilde{\nabla}^{2}=\frac{d^{2}}{d \eta^{2}}+\frac{1}{\eta} \frac{d}{d \eta}-k^{2} .
$$

За нестисливості в рівняннях (8)-(12) необхідно прийняти:

$$
\vartheta=0 .
$$

Відповідно система (15)-(18) має вигляд:

$$
\begin{gathered}
\left(\tilde{\nabla}^{2}-i \omega \beta\right) U_{\xi}+i k \beta Q=0, \\
{\left[\tilde{\nabla}^{2}-\left(\frac{1}{\eta^{2}}+i \omega \beta\right)\right] U_{\eta}-\beta \frac{d Q}{d \eta}=0,} \\
\frac{\partial U_{\eta}}{d \eta}+\frac{1}{\eta} U_{\eta}-i k U_{\xi}=0 .
\end{gathered}
$$

3 рівнянь (21)-(23) випливає, що

$$
\left[\tilde{\tilde{\nabla}}^{2}-\left(\frac{1}{\eta^{2}}+i \omega \beta\right)\right]\left[\tilde{\tilde{\nabla}}^{2}-\frac{1}{\eta^{2}}\right] U_{\eta}=0 .
$$

Розв'язок рівняння (24), яке $\epsilon$ кінцевим при $\eta=0$, має також вигляд

$$
U_{\eta}=B_{1} J_{1}\left(C_{1} \eta\right)+B_{2} J_{1}\left(C_{2} \eta\right) .
$$

де тепер:

$$
\begin{gathered}
C_{1}^{2}=-k^{2}-i \beta \omega, \\
C_{2}^{2}=-k^{2} .
\end{gathered}
$$

3 рівняння (23) і співвідношення (25) виходить, що компонента швидкості $U_{\xi}$ має також вигляд

$$
U_{\xi}=A_{1} J_{0}\left(C_{1} \eta\right)+A_{2} J_{0}\left(C_{2} \eta\right),
$$

причому, в цьому випадку у співвідношеннях

$$
\begin{array}{r}
B_{1}=m_{1} A_{1}, B_{2}=m_{2} A_{2}, \\
\text { потрібно взяти } \quad m_{1}=\frac{i k}{C_{1}}, m_{2}=\frac{i k}{C_{2}} .
\end{array}
$$

3 граничних умов при $\eta=1$

$$
U_{\xi}=0, U_{\eta}=0 .
$$

Отримуємо:

$$
\begin{gathered}
A_{1} J_{0}\left(C_{1}\right)+A_{2} J_{0}\left(C_{2}\right)=0, \\
m_{2} A_{1} J_{1}\left(C_{1}\right)+m_{2} A_{2} J_{1}\left(C_{2}\right)=0 .
\end{gathered}
$$

Для того, щоб система (32), (33) мала розв'язки, повинно задовольнятись характеристичне рівняння

$$
m_{1} J_{0}\left(C_{2}\right) J_{1}\left(C_{1}\right)-m_{2} J_{0}\left(C_{1}\right) J_{1}\left(C_{2}\right)=0 .
$$

Якщо розглядати тільки низькочастотні розв'язки та ввести припущення:

$$
\begin{gathered}
\left|\frac{\beta}{\omega}\right|>>1 \text { або }|\beta|>>|\omega|, \\
|\omega \beta|>>\left|\kappa^{2}\right|,
\end{gathered}
$$

то співвідношення $(26,27,30)$ набувають вигляду:

$$
\begin{gathered}
C_{1}^{2}=-i \beta \omega, C_{2}^{2}=-k^{2} \\
m_{1}=\frac{i k}{C_{1}}, m_{2}=1 .
\end{gathered}
$$

Відповідно компоненти швидкості, тиску і характеристичного рівняння мають вигляд: 


$$
\begin{gathered}
U_{\xi}=A_{1}\left[J_{0}\left(C_{1} \eta\right)-J_{0}\left(C_{1}\right)\right], \\
U_{\eta}=i k A_{1}\left[\frac{J_{1}\left(C_{1} \eta\right)}{J_{1} C_{1}}-\frac{J_{0}\left(C_{1}\right) \eta}{2}\right], \\
Q=-\frac{\omega A_{1}}{k} J_{0}\left(C_{1}\right), \\
k\left[2 J_{1}\left(C_{1}\right)-C_{1} J_{0}\left(C_{1}\right)\right]=0 .
\end{gathered}
$$

3 рівняння (42) випливає, що

$$
k=0 .
$$

3 огляду на рівність (43) із співвідношення (40) отримуємо, що

$$
U_{\eta}=0 \text {. }
$$

Звідси бачимо, що у випадку нестисливої рідини при виведенні спрощеної моделі припущення $U_{\eta}=0$ є цілком обгрунтованим.

Якщо враховувати порядок складових в рівнянні (21)-(23) за співвідношеннями (35), (36), (39), (44), їх можна спростити до вигляду:

$$
\begin{gathered}
\left(\frac{d^{2}}{d \eta^{2}}+\frac{1}{\eta} \frac{d}{d \eta}-i \omega \beta\right) U_{\xi}+i k \beta Q=0, \\
\frac{d Q}{d \eta}=0 .
\end{gathered}
$$

Відповідне вихідне рівняння для опису руху нестисливої рідини в циліндричній трубі має загальновідомий вигляд

$$
\frac{\partial^{2} u_{\xi}}{\partial \eta^{2}}+\frac{1}{\eta} \frac{\partial u_{\xi}}{\partial \eta}-\beta \frac{\partial u_{\xi}}{\partial \tau}-\beta \frac{\partial q}{\partial \xi}=0 .
$$

Тут допускається, що

$$
\frac{\partial q}{\partial \eta}=0
$$

Обговорення отриманих результатів. Розв'язок рівнянь для опису руху нестисливої рідини в циліндричній трубі, використовуючи модель із врахуванням дисипативних процесів течії в'язкої рідини, дало змогу отримати розподіл полів швидкостей із задовільною похибкою для інженерних розрахунків.

Проведені експериментальні дослідження неусталених потоків [15] рідини в циліндричних трубопроводах дали змогу отримати розподіл швидкостей, які підтвердили відповідність отриманих розрахунків на основі дисипативної моделі.

Визначено перспективні напрями досліджень, які уможливлять подальший розвиток пропонованого підходу для важливих практичних задач.

Висновки. Розв'язано задачу неусталених рухів рідини в трубах для визначення розподілу швидкостей рідини в перерізах трубопроводу в осьовому та радіальному напрямках. Запропоновано удосконалену ме-

тодику розрахунку неусталених потоків для нестисливої рідини на основі дисипативної моделі. 3'ясовано, що в цьому випадку припущення про нехтування компонентою радіальної швидкості $€$ асимптотично обгрунтованим.

Використана модель із врахуванням дисипативних процесів течії в'язкої рідини, яку досліджували варіаційним методом 3 урахуванням початкових і граничних умов, дає змогу отримати розподіл полів швидкостей. Наведено низькочастотні розв'язки рівнянь Нав'єСтокса для спрощеної моделі нестисливої рідини.

\section{References}

Adamkowski, A., \& Lewandowski, M. (2006). Experimental examination of unsteady friction models for transient pipe flow simulation. Trans. ASME. J. Fluids Eng., 128(6), 1351-1363.

Barmetov, Iu. P., \& Palishkin, D. A. (2007). Matematicheskie modeli gidravlicheskogo udara. Materialy 45 Otchetnoi nauchnoi konferentcii za 2006 god Voronezhskoi gosudarstvennoi tekhnologicheskoi akademii. (Part 2), (pp. 106-107). Voronezh: VGTA. [In Russian].

Bondarenko, N. I., \& Terentev, Iu. I. (2009). O neustanovivshemsia dvizhenii szhimaemoi zhidkosti v napornom truboprovode. Moscow: Moscow State Technical University, 54 p. Dep. v VINITI RAN 15.10.2009, № 620-V2009. [In Russian].

Charnyi, I. A. (1975). Neustanovivsheesia dvizhenie realnoi zhidkosti $v$ trubakh. Moscow: Nedra, 296 p. [In Russian].

D'Suza, A. F., \& Oldenburger, R. (1964). Dinamicheskaia kharakteristika gidravlicheskikh truboprovodov. Teor. osn. inzh. rasch., 3, 196-205. [In Russian].

Girgidov, A. D. (2009). O dissipatcii energii pri dvizhenii neszhimaemoi zhidkosti. Dokl. RAN. 426(5), 626-628. [In Russian].

Gnativ, R. M. (2013). Doslidzhennia rozpodilu shvidkostei pri neustalenii techiï ridini v truboprovodi. Promislova gidravlika i pnevmatika, 2(40), 57-59. [In Ukrainian].

Gnativ, R. M., \& Mikitin, M. I. (2012). Asimptotichnii metod vivedennia disipativnoï modeli neustalenogo potoku ridini. Promislova gidravlika i pnevmatika, 1(35), 40-44. [In Ukrainian].

Gromeka, I. S. (1952). K teorii dvizheniia zhidkosti v uzkikh tcilindricheskikh trubakh. Sobr. soch. AN SSSR, 149-171. [In Russian].

Jayasinghe, D. A. P., \& Leutheusser, H. J. (1972). Pulsatile Waterhammer Subject to Laminar Friction. J. Fluids Eng, 94(2), 467-472.

Magrakvelidze, T. (2005). K voprosu raspredeleniia skorostei pri turbulentnom techenii zhidkosti v krugloi trube. Sb. trudov In-t sistem upr. AN Gruzii, 9, 96-101. [In Russian].

Mochalin, E. V. (2002). Variatcionnaia formulirovka zadachi o prostranstvennom dvizhenii neszhimaemoi zhidkosti. Sobr. nauchn. trudov DGMI, 15, 269-280. Alchevsk: DGMI. [In Russian].

Popov, D. N. (1977). Dinamika i regulirovanie gidro- i pnevmosistem. Moscow: Mashinostr., pp. 185-249. [In Russian].

Rakhmatullin, Sh. I., \& Kim, D. P. (2006). Vliianie stepeni turbulentnosti i chastoty turbulentnykh pulsatcii na gidravlicheskoe soprotivlenie krugloi truby. Neft. kh-vo, 11, 110-111. [In Russian].

Shablovskii, O. N. (2007). Neklassicheskie dissipativnye protcessy v neszhimaemoi zhidkosti. Fundam. fiz.-mat. probl. i modelir. tekhn.tekhnol. sistem, 10, 96-100. [In Russian].

O. M. Yahno', R. M. Hnativ', I. R. Hnativ

${ }^{I}$ National Technical University of Ukraine "Igor Sikorsky Kyiv Polytechnic Institute", Kyiv, Ukraine ${ }^{2}$ Lviv Polytechnic National University, Lviv, Ukraine ${ }^{3}$ Lviv National Agrarian University, Dublyany, Ukraine

\section{MATHEMATICAL MODEL FOR THE STUDY OF UNSTEADY FLOWS OF AN INCOMPRESSIBLE FLUID IN PIPES}

The authors have analysed some scientific works on solving problems of non-stationary movement of fluid in cylindrical tubes. It has been established that when solving problems of unsteady fluid motions in pipes, it becomes necessary to determine fluid velocities in pipeline sections both in axial and radial directions. Classical methods for solving this problem do not give satisfactory results. The purpose of the research is to improve the method of calculating unsteady fluid flows based on a dissipative model. The studies 
used a model with allowance for dissipative flow of a viscous fluid, which was studied by the variational method with regard to the initial and boundary conditions. The object of the research is hydraulic processes in unsteady viscous fluid flows in a cylindrical pipeline. The paper proposes an improved method for calculating unsteady flows for an incompressible fluid based on a dissipative model. We have found that in this case the assumption that the radial velocity component is neglected is asymptotically justified. Lowfrequency solutions of the Navier-Stokes equations for a simplified model of an incompressible fluid are given. The dissipative model is based on two assumptions about the order of the solutions of the Navier-Stokes equations for time and axial coordinate. In this case, assumptions about the order of magnitude of the components of speed are not displayed. The solution of equations for describing the motion of incompressible fluid in a cylindrical tube using a model concerning the dissipative processes of the viscous fluid flow allowed obtaining the distribution of velocity fields with a satisfactory error for engineering calculations. Prospective areas of the research were identified. These areas would enable further development of the proposed approach for important practical tasks.

Keywords: unsteady; non-stationary; fluid motion; dissipative model; velocity distribution. 